OCU-PHYS-379, AP-GR-104, YITP-12-101

\title{
Ultra-high energy collision with neither black hole nor naked singularity
}

\author{
${ }^{1}$ Ken-ichi Nakao* 2 Masashi Kimura ${ }^{\dagger},{ }^{3}$ Mandar Patil ${ }^{\ddagger},{ }^{3}$ Pankaj S. Joshi ${ }^{\S}$ \\ ${ }^{1}$ Department of Mathematics and Physics, Graduate School of Science, \\ Osaka City University, Osaka 558-8585, Japan. \\ ${ }^{2}$ Yukawa Institute for Theoretical Physics, \\ Kyoto University, Kyoto 606-8502, Japan. \\ ${ }^{3}$ Tata Institute of Fundamental Research, \\ Homi Bhabha Road, Mumbai 400005, India.
}

\begin{abstract}
We explore the collision between two concentric spherical thin shells. The inner shell is charged, whereas the outer one is either neutral or charged. In the situation we consider, the charge of the inner shell is larger than its gravitational mass, and the inside of it is empty and regular. Hence the domain just outside it is described by the overcharged Reissner-Nordström geometry whereas the inside of it is Minkowski. First, the inner shell starts to shrink form infinity with finite kinetic energy, and then the outer shell starts to shrink from infinity with vanishing kinetic energy. The inner shell bounces on the potential wall and collides with the ingoing outer shell. The energy of collision between these shells at "their center of mass frame" does not exceed the total energy of the system. By contrast, by virtue of the very large gamma factor of the relative velocity of the shells, the energy of collision between two of the constituent particles of these shells at their center of mass frame can be much larger than the Planck scale. This result suggests that the black hole or naked singularity is not necessary for ultra-high energy collision of particles.
\end{abstract}

PACS numbers: 04.20.Dw, 04.70.-s, 04.70.Bw

\footnotetext{
* Electronic address: knakao@sci.osaka-cu.ac.jp

$\dagger$ Electronic address: mkimura@yukawa.kyoto-u.ac.jp

$\ddagger$ Electronic address: mandarp@tifr.res.in

$\S$ Electronic address: psj@tifr.res.in
} 


\section{INTRODUCTION}

Banados, Silk and West (BSW) recently pointed out that the Kerr black hole can act as a particle accelerator[1]: Two particles dropped in from infinity at rest, traveling along the timelike geodesics can collide with arbitrarily large energy at their center of mass frame, if the Kerr black hole is extremely spinning and orbital angular momentum of one of the particles takes a specific fine-tuned value. Then, the possible astrophysical implications of this process around the event horizon of the central supermassive black hole in the context of annihilations of the dark matter particles accreted from the galactic halo were investigated [2]. The BSW process of particle acceleration suffers from several drawbacks and limitations pointed out in [3] : such as for example, a fine-tuning of the orbital angular momenta of the particles, a neglect of the self-gravity of particles in their analysis, and an upper bound on the spin of the Kerr black hole formed in our universe [4]. There were many investigations of this acceleration mechanism in the background of Kerr as well as many other black holes [5].

Two of the present authors, MP and PSJ (PJ), pointed out the other possibility. Particle collisions with arbitrarily large energy at the center of mass frame may occur in the naked singular Kerr spacetime if the total angular momentum of the central naked singularity is very close to the critical value[6]. This mechanism is physically rather different from the BSW process. In the case of the BSW process, one of the two particles asymptotically approaches the event horizon by virtue of its special value of the orbital angular momentum. Since the event horizon is generated by the outward null geodesics, the world line of the particle asymptotically becomes outward null. Hence, the relative velocity between this particle and another particle falling to the Kerr black hole can be very large. As a result, the center of mass energy of the collision between these particles can be arbitrarily large. By contrast, in the case of the PJ process, one of the two particles falls inward but eventually turns to outward due to the repulsive nature of the naked singularity or due to the centrifugal potential. Then, it can collide with another particle falling inward. In this mechanism, the large relative velocity between these two particles at the collision event can be achieved by virtue of the deep gravitational potential of the almost extreme naked singular geometry.

Like the BSW process, the PJ process of particle acceleration also would have certain drawbacks and limitations. As in the case of BSW's analysis, the self-gravities of the particles are neglected in PJ's analysis, and it is unclear whether the naked singular Kerr geometry 
appears in our universe[7]. However, in the case of the PJ process, no fine-tuning of the orbital angular momenta of the colliding particles is necessary.

The issue of the self-gravity of the point particles is difficult to deal with in general. If the effects of self-gravity and gravitational radiation are important in a collision between elementary particles, such a collision process must be described by the quantum gravity: The gravitational interaction between elementary particles is to be necessarily quantum in nature. Hence, even if BSW or PJ analysis does not predict accurately the high energy collision of the elementary particles, their result implies that collisions of elementary particles with the center of mass energy high enough to cause quantum gravitational interactions may occur in the Kerr spacetime. However, it is a fascinating primary question what happens when the center of mass energy of a collision becomes comparable to the total mass of the system within the framework of general relativity.

Unfortunately, it is very difficult to treat analytically the motion of matter in the Kerr spacetime, if we will take into account the effect of their self-gravity. Hence, it is worthwhile to notice that the similar processes to both of the BSW and PJ processes may occur in the Reissner-Nordström spacetime [8-10]: it is much easier than the case of the Kerr spacetime to treat analytically the motion of matter in the Reissner-Nordström spacetime by virtue of its spherical symmetry. In the case of a process similar to the BSW one, a particle with charge of the same sign as that of the extreme Reissner-Nordström black hole radially falls toward the black hole from infinity. If the charge of the particle is equal to its mass, the particle asymptotically approaches the event horizon. After the charged particle starts to fall, another neutral particle also starts a radial fall toward the black hole from infinity. Then, it eventually overtakes and collides with the charged particle previously falling. The closer to the event horizon the collision event is, the higher will be its energy at their center of mass frame, and arbitrarily large collision energy is in principle possible. By contrast, in the case of the process similar to PJ's one, two radially moving neutral particles can collide with arbitrarily large energy at their center of mass frame. One of the two particles falls inward from infinity and eventually turns back outwards due to the repulsive nature of the central naked singularity. Then it collides with another particle which starts to fall towards the naked singularity after the first particle have started. The very large collision energy is possible due to the deep gravitational potential of almost extreme central naked singularity, like in the case of the naked singular Kerr spacetime. 
A system of concentric spherical shells with infinitesimal width in the Reissner-Nordström spacetime is very useful to study the effect of the self-gravity in the BSW and PJ processes, since their dynamical degrees of freedom are finite and hence the system is tractable analytically. The stress-energy tensor diverges on the shells, since finite energy and momentum concentrate on the infinitesimally thin domains. This means that these shells are categorized into the so-called curvature polynomial singularity[11] through the Einstein equations. Since each shell has finite mass and momentum, the volume integral of the stress-energy tensor is finite: the components of the stress-energy tensor are distributional sources of Einstein's equations. Here we should note that the distributional source is a technical simplification usually adopted in theoretical study of gravitational physics. For example, in the framework of Newtonian gravity, massive point particles are useful idealization to study the celestial mechanics, although real stars have finite sizes. By this idealization, the dynamical degrees of freedom of the system become finite, and as a result, the analysis is very easy. If the size of each star is much smaller than the size of the system, this point-particle approximation will give sufficiently accurate prediction about their orbits. The divergences of the gravitational potential and the gravitational force just at the point particle are artificial due to this technical idealization, and the gravity of each star is assumed to be so small that the Newtonian approximation is valid. By contrast, in the framework of general relativity, the point-particle approximation is impossible, since the point particle is so seriously singular that the spacetime metric cannot be defined on it. However the thin-shell approximation is possible even in the framework of general relativity, since the metric is defined on the infinitesimally thin shell even though the stress-energy tensor diverges on it. We can derive the equation of motion for the shell which is consistent with the Einstein equations by the so-called Israel formalism[12, 13]. If the shell is highly symmetric (e.g. spherically symmetric), the degrees of freedom becomes finite, and as a result, the analysis is very easy. The divergence of the Ricci tensor at the shell is artificial due to this technical simplification. The thin-shell approximation is valid if the width of the shell is much smaller than the size of the system.

Two of the present authors, MK, KN, and their collaborator, Tagoshi, studied the collision between two concentric spherical dust shells in the Reissner-Nordström black hoke geometry; one of the two shells has a charge, whereas another is neutral. This example corresponds to the spherical shell version of the BSW process. By virtue of the spherical symmetry, they 
treated this system exactly and showed that the effect of the self-gravity makes the collision energy finite as long as we focus on the collision event observable for distant observers. Then, the present authors investigated the collision between two concentric neutral spherical dust

shells in the naked singular Reissner-Nordström geometry[10]: This example corresponds to the spherical shell version of the PJ process. They showed that the upper bound on the energy of the collision between the shells appears due to the self-gravity of the shells also in this case as long as we focus on the collision event observable for the distant observers. But, the energy of the collision between two of the constituent particles of these shells can still exceed the Planck scale. Furthermore, in the case of the naked singular geometry, the timescale to occur the high-energy collision may be much shorter than the case that corresponds to the BSW process[10].

In this paper, we investigate a collision between two concentric infinitesimally thin spherical shells made up of dust particles in a situation of no black hole and no naked singularity, taking into account their self-gravity exactly. The domain inside the inner shell is described by the Minkowskian geometry, whereas the outside of it is described by the over-charged Reissner-Nordström geometry. The outer shell may or may not have charge, but the outside of it is assumed to be also described by the over-charged Reissner-Nordström geometry. Even in this situation, very high energy collision between two of the constituent particles of these shells through a similar mechanism to the PJ process may occur, since the domain between these shells is described by the naked singular Reissner-Nordström geometry.

This paper is organized as follows. In Sec. II, we briefly review Israel's formalism. Also in this section, we show the situation we consider and derive equations of motion for spherical shells. In Sec. III, the collision energy of two of constituent particles of the shells at their center of mass frame is shown. Sec. IV is devoted to summary and discussion.

In this paper, we adopt the geometrized unit in which the speed of light and Newton's gravitational constant are unity.

\section{EQUATION OF MOTION FOR SPHERICAL SHELLS}

We consider two concentric spherical shells which are infinitesimally thin. These shells may have $U(1)$ charge (see Fig,1).

The trajectories of these shells in the spacetime are timelike hypersurfaces: the inner 


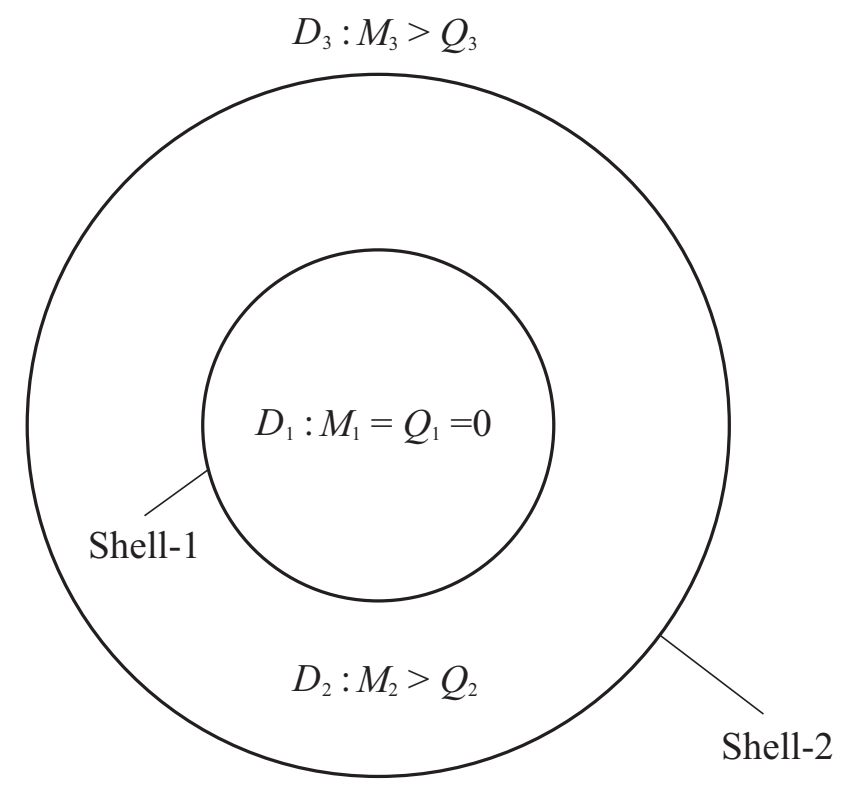

FIG. 1: This is a snapshot of the spherically symmetric spacetime divided into two domains $D_{1}$, $D_{2}$ and $D_{3}$ by the trajectories of the shell-1 and shell-2, i.e., $\Sigma_{1}$ and $\Sigma_{2}$.

hypersurface is denoted by $\Sigma_{1}$, whereas the outer hypersurface is denoted by $\Sigma_{2}$. Correspondingly, the inner shell is called the shell-1, whereas the outer shell is called the shell-2. $\Sigma_{1}$ and $\Sigma_{2}$ divide the spacetime into three domains: the innermost domain is denoted by $D_{1}$, the middle one is denoted by $D_{2}$, and the outermost one is denoted by $D_{3}$. By the symmetry of this system, the geometry of the domain $D_{i}(i=1,2,3)$ is described by the Reissner-Nordström solution whose line element is given by

$$
d s^{2}=-f_{i}(r) d t_{i}^{2}+\frac{1}{f_{i}(r)} d r^{2}+r^{2}\left(d \theta^{2}+\sin ^{2} \theta d \phi^{2}\right)
$$

with

$$
f_{i}(r)=1-\frac{2 M_{i}}{r}+\frac{Q_{i}^{2}}{r^{2}},
$$

where $M_{i}$ is the mass parameter and $Q_{i}$ is the $U(1)$ charge within the sphere of the radius $r$. We should note that the coordinate $t_{i}$ is not continuous across the shells, whereas $r, \theta$ and $\phi$ are everywhere continuous. The $U(1)$ gauge field in the domain $D_{i}$ is given by

$$
A_{\mu}=\frac{Q_{i}}{r} \delta_{\mu}^{t}
$$

In the Reissner-Nordström spacetime of the mass parameter $M$ and the charge $Q$, there is a spacetime singularity at $r=0$. This singularity is timelike and thus is necessarily 
locally naked. The location of the horizon in the Reissner-Nordström spacetime is given by a positive root of the equation $f(r) \equiv 1-2 M / r+Q^{2} / r^{2}=0$. There are two roots to this equation given by

$$
r=r_{ \pm} \equiv M \pm \sqrt{M^{2}-Q^{2}} .
$$

There are two real positive roots of the equation if $M>Q$. The larger root $r=r_{+}$is the location of the event horizon and this spacetime contains a spherically symmetric charged black hole. The smaller root $r=r_{-}$corresponds to the Cauchy horizon associated with the timelike singularity at $r=0$. If $M=Q$, there is only one positive root. In this case, the black hole has a degenerate event horizon at $r=M=Q$; we call this the extreme black hole. In the case of $M<Q$, there is no real root of the equation $f(r)=0$. Thus, the event horizon is absent and the timelike singularity at $r=0$ is exposed to the asymptotic observer at infinity. This configuration thus contains a globally visible naked singularity.

As mentioned, even though $\Sigma_{A}(A=1,2)$ are spacetime singularities, we can derive the equation of motion for each spherical shell which is consistent with the Einstein equations by the so-called Israel formalism[12, 13]. Let us cover the neighborhood of one singular hypersurface $\Sigma_{A}$ by a Gaussian normal coordinate $\lambda$, where $\partial / \partial \lambda$ is perpendicular to $\Sigma_{A}$ and directs from $D_{A}$ to $D_{A+1}$. Then, the sufficient condition to apply Israel's formalism is that the stress-energy tensor is written in the form

$$
T_{\mu \nu}=\sum_{A} S_{\mu \nu}^{(A)} \delta\left(\lambda-\lambda_{A}\right)
$$

where $\Sigma_{A}$ is located at $\lambda=\lambda_{A}, \delta(x)$ is Dirac's delta function, and $S_{\mu \nu}^{(A)}$ is finite and called the surface stress-energy tensor of $\Sigma_{A}$.

The junction condition of the metric tensor is given as follows. We impose that the metric tensor $g_{\mu \nu}$ is continuous across $\Sigma_{A}$, but its derivative is not necessarily so. The unit normal vector to $\Sigma_{A}$ is $\partial / \partial \lambda$. Hereafter, we denote it by $n^{\mu}$. The intrinsic metric of $\Sigma_{A}$ is given by

$$
h_{\mu \nu}=g_{\mu \nu}-n_{\mu} n_{\nu} .
$$

Then, the extrinsic curvature is defined by

$$
K_{\mu \nu}^{(i)}=h^{\alpha}{ }_{\mu} h^{\beta}{ }_{\nu} \nabla_{\alpha}^{(i)} n_{\beta},
$$

where $\nabla_{\alpha}^{(i)}$ is the covariant derivative with respect to the metric in the domain $D_{i}$. This extrinsic curvature describes how $\Sigma_{A}$ is embedded into the domain $D_{i}$. In accordance with 
Israel's formalism, the Einstein equations lead to

$$
K_{\mu \nu}^{(A+1)}-K_{\mu \nu}^{(A)}=8 \pi\left(S_{\mu \nu}^{(A)}-\frac{1}{2} h_{\mu \nu} \operatorname{tr} S^{(A)}\right) .
$$

In this article, the surface stress-energy tensors of the shells are assumed to be that of pressure-less matter, i.e., the dust

$$
S_{\mu \nu}^{(A)}=\sigma_{A} u_{\mu} u_{\nu}
$$

where $\sigma_{A}$ is the energy per unit area on $\Sigma_{A}$, and $u^{\mu}$ is the 4 -velocity. We assume that $\sigma_{A}$ is positive. By the spherical symmetry, the motion of the shell- $A$ is described in the form $t_{i}=t_{i}(\tau)$ and $r=r(\tau)$, where $i=A$ or $i=A+1$, and $\tau$ is the proper time of the shell- $A$. The 4-velocity is given by

$$
u^{\mu}=\left(\dot{t}_{i}, \dot{r}, 0,0\right) \text {, }
$$

where a dot means a derivative with respect to $\tau$. Then, $n_{\mu}$ is given by

$$
n_{\mu}=\left(-\dot{r}, \dot{t}_{i}, 0,0\right) \text {. }
$$

Together with $u^{\mu}$ and $n^{\mu}$, the following unit vectors form an orthonormal frame;

$$
\begin{aligned}
e_{(\theta)}^{\mu} & =\left(0,0, \frac{1}{r}, 0\right), \\
e_{(\phi)}^{\mu} & =\left(0,0,0, \frac{1}{r \sin \theta}\right) .
\end{aligned}
$$

The extrinsic curvature is obtained as

$$
\begin{aligned}
K_{\mu \nu}^{(i)} u^{\mu} u^{\nu} & =\frac{1}{f_{i} \dot{t}_{i}}\left(\ddot{r}+\frac{f_{i}^{\prime}}{2}\right), \\
K_{\mu \nu}^{(i)} e_{(\theta)}^{\mu} e_{(\theta)}^{\nu} & =K_{\mu \nu}^{(i)} e_{(\phi)}^{\mu} e_{(\phi)}^{\nu}=-n^{a} \partial_{a} \ln r=-\frac{f_{i}}{r} \dot{t}_{i}
\end{aligned}
$$

and the other components vanish, where a prime means a derivative with respect to $r$. By the normalization condition $u^{\mu} u_{\mu}=-1$, we have

$$
\dot{t}_{i}= \pm \frac{1}{f_{i}} \sqrt{\dot{r}^{2}+f_{i}} \text {. }
$$

We assume that there is no black hole initially, or equivalently, $f_{i}$ is initially everywhere positive. Then, $t_{i}$ corresponds to the time coordinate and its derivative $\dot{t}_{i}$ should be positive initially. Thus, we should choose the plus sign in the right hand side of Eq. (3). If the shell- $A$ enters into a black hole and $f_{i}$ becomes negative, $t_{(i)}$ becomes spatial coordinate. If so, $\dot{t}_{(i)}$ may change its sign, and hence there is a possibility that we have to choose the minus sign in Eq. (3). But, as long as we do not mention, hereafter we assume the plus sign in Eq. (3). 


\section{A. The effective potential}

From $t-t$ and $\theta-\theta$ components of Eq. (2), we obtain the following relation.

$$
\sqrt{\dot{r}^{2}+f_{A+1}(r)}-\sqrt{\dot{r}^{2}+f_{A}(r)}=-\frac{m_{A}}{r},
$$

with

$$
m_{A}:=4 \pi \sigma_{A} r^{2}=\text { constant. }
$$

Note that $m_{A}$ is positive, since $\sigma_{A}$ is assumed to be positive.

Let us rewrite Eq. (4) into the form of the energy equation. First, we write it in the form

$$
\sqrt{\dot{r}^{2}+f_{A+1}(r)}=\sqrt{\dot{r}^{2}+f_{A}(r)}-\frac{m_{A}}{r},
$$

and then take a square of its both sides:

$$
\dot{r}^{2}+f_{A+1}(r)=\dot{r}^{2}+f_{A}(r)+\left(\frac{m_{A}}{r}\right)^{2}-\frac{2 m_{A}}{r} \sqrt{\dot{r}^{2}+f_{A}(r)} .
$$

Furthermore, we rewrite the above equation in the form

$$
\sqrt{\dot{r}^{2}+f_{A}(r)}=\frac{r}{2 m_{A}}\left[f_{A}(r)-f_{A+1}(r)+\left(\frac{m_{A}}{r}\right)^{2}\right] .
$$

By taking a square of the both sides of the above equation, we have

$$
\dot{r}^{2}+V_{A}(r)=0
$$

where

$$
V_{A} \equiv f_{A}(r)-\left(\frac{r}{2 m_{A}}\right)^{2}\left[f_{A}(r)-f_{A+1}(r)+\left(\frac{m_{A}}{r}\right)^{2}\right]^{2} .
$$

The above equation is regarded as the energy equation for the shell- $A$. The function $V_{A}(r)$ corresponds to the effective potential. In the domain allowed for the motion of the shell- $A$, $V_{A} \leq 0$ should be satisfied.

Here note that the negativity of the right hand side of Eq. (44) implies to $f_{A+1}<f_{A}$ in the domain allowed for the motion of the shell- $A$. Furthermore, since the left hand side of Eq. (5) is non-negative, the right hand side of it should also be non-negative. Substituting Eq. (7) into the right hand side of Eq. (5), we find that the following inequality should be satisfied in the allowed domain;

$$
F_{A}(r) \equiv f_{A}(r)-f_{A+1}(r)-\left(\frac{m_{A}}{r}\right)^{2}=\frac{2 m_{A} \mathcal{E}_{A}}{r}-\frac{Q_{A+1}^{2}-Q_{A}^{2}+m_{A}^{2}}{r^{2}} \geq 0,
$$


where

$$
\mathcal{E}_{A} \equiv \frac{M_{A+1}-M_{A}}{m_{A}}
$$

We should note that $\mathcal{E}_{A}$ is not necessarily positive even in the case of $m_{A}>0$, since $M_{A+1}-$ $M_{A}$ does not necessarily represent the energy of the shell- $A[17]$.

\section{B. The allowed domain for the motion of the shells}

In the case of $\mathcal{E}_{A}>0$, Eq. (10) leads to

$$
r \geq \rho_{A}
$$

where

$$
\rho_{A} \equiv \frac{Q_{A+1}^{2}-Q_{A}^{2}+m_{A}^{2}}{2 \mathcal{E}_{A} m_{A}} .
$$

In the case of $\mathcal{E}_{A}=0$, Eq. (10) leads to

$$
Q_{A+1}^{2}-Q_{A}^{2}+m_{A}^{2} \leq 0
$$

In the case of $\mathcal{E}_{A}<0$, Eq. (10) leads to

$$
r \leq \rho_{A}
$$

Since $r$ is positive, the condition

$$
Q_{A+1}^{2}-Q_{A}^{2}+m_{A}^{2}<0
$$

should hold so that Eq. (13) has a solution [18].

Here we should note that there is a possibility that if the shell- $A$ is in the domain with $Q_{A+1}>M_{A+1}$, there is a possibility that the domain with $V_{A}<0$ is not allowed for the motion of the shell- $A$. We see such a case in the next section.

In this article, we assume $M_{1}=0=Q_{1}, M_{2}<Q_{2}$ and $M_{3}<Q_{3}$; the shell-1 is charged, whereas the shell-2 is not necessarily charged. The domain $D_{1}$ is then described by the Minkowski geometry, whereas $D_{2}$ and $D_{3}$ are described by the over-charged ReissnerNordström geometry. Hence $f_{A+1}(A=1,2)$ is everywhere positive. We can see that

$$
V_{A}\left(\rho_{A}\right)=f_{A+1}\left(\rho_{A}\right)>0
$$

The above result implies that $r=\rho_{A}$ is necessarily in the domain forbidden for the motion

of the shell- $A$. By the assumption on $M_{i}$ and $Q_{i}(i=1,2,3)$, since the left hand side of Eq.(51) is positive, the function $F_{A}(r)$ (10) takes positive value. 


\section{THE ENERGY OF COLLISION BETWEEN TWO CONCENTRIC SHELLS}

In accordance with Ref. [9, 10], the energy $E_{\mathrm{cm}}$ of the collision between the shells in the "center of mass frame" is given by

$$
\begin{aligned}
E_{\mathrm{cm}}^{2} & =-g_{\mu \nu}\left(m_{1} u_{1}^{\mu}+m_{2} u_{2}^{\mu}\right)\left(m_{1} u_{1}^{\nu}+m_{2} u_{2}^{\nu}\right) \\
& =m_{1}^{2}+m_{2}^{2}+\frac{2 m_{1} m_{2}}{f_{2}}\left[\sqrt{\left(f_{2}-V_{1}\right)\left(f_{2}-V_{2}\right)}+\sqrt{V_{1} V_{2}}\right],
\end{aligned}
$$

where we have assumed that the sign of $u_{1}^{r}$ is pooisite to that of $u_{2}^{r}$. We are also interested in the energy $E_{\mathrm{P}}$ of the collision between two of constituent particles of these shells in their center of mass frame. We assume that all constituent particles have an identical mass $m$. Then, we obtain

$$
\begin{aligned}
E_{\mathrm{P}}^{2} & =-g_{\mu \nu} m^{2}\left(u_{1}^{\mu}+u_{2}^{\mu}\right)\left(u_{1}^{\nu}+u_{2}^{\nu}\right) \\
& =2 m^{2}\left[1+\frac{\sqrt{\left(f_{2}-V_{1}\right)\left(f_{2}-V_{2}\right)}+\sqrt{V_{1} V_{2}}}{f_{2}}\right] .
\end{aligned}
$$

From the above equation, we can expect that $E_{\mathrm{cm}}$ and $E_{\mathrm{P}}$ are large if the collision occurs at the minimum of $f_{2}$. The function $f_{2}(r)$ takes a minimum value at

$$
r=r_{\min } \equiv \frac{Q_{2}^{2}}{M_{2}}
$$

and the minimum value $f_{2}\left(r_{\min }\right)$ is equal to $1-\left(M_{2} / Q_{2}\right)^{2}$. If $Q_{2}$ is very close to $M_{2}$, the minimum value $f_{2}\left(r_{\mathrm{min}}\right)$ is very small and $E_{\mathrm{cm}}$ and $E_{\mathrm{P}}$ may be very large. We shall estimate how large $E_{\mathrm{cm}}$ and $E_{\mathrm{P}}$ of the collision at $r=r_{\text {min }}$ can be. For this purpose, we parametrize $Q_{2}$ as

$$
Q_{2}=(1+\epsilon) M_{2}
$$

and we assume $0<\epsilon \ll 1$.

\section{A. The shell-1}

As mentioned, we assume both $M_{1}$ and $Q_{1}$ vanish, and hence we have $Q_{2}^{2}-Q_{1}^{2}+m_{1}^{2}=$ $Q_{2}^{2}+m_{1}^{2}>0$. This implies that $\mathcal{E}_{1}$ should be positive, and, as a result, $M_{2}$ should also be 
positive: If $\mathcal{E}_{1}$ is negative, the areal radius $r$ should be less than zero by Eq. (13), but this is not the case of our interest. Since we assume $Q_{2}>M_{2}, Q_{2}$ is necessarily positive.

The effective potential of the shell-1 is written in the form

$$
V_{1}(r)=1-\mathcal{E}_{1}^{2}+\frac{\mathcal{E}_{1}}{m_{1} r}\left(Q_{2}^{2}-m_{1}^{2}\right)-\left(\frac{Q_{2}^{2}-m_{1}^{2}}{2 m_{1} r}\right)^{2}
$$

The roots of $V_{1}(r)=0$ are

$$
r=\rho_{ \pm} \equiv \frac{1}{2 m_{1}\left(\mathcal{E}_{1} \mp 1\right)}\left(Q_{2}^{2}-\frac{M_{2}^{2}}{\mathcal{E}_{1}^{2}}\right) .
$$

By some consideration, we see that the motion of the shell-1 is necessarily unbound $\mathcal{E}_{1}>1$ and its allowed domain is $r \geq \rho_{+}$, as long as we assume $Q_{2}>M_{2}$. The detail of the consideration is given in Appendix $\mathrm{A}$.

In order that the collision occurs at $r=r_{\min }, r=r_{\min }$ should be in a domain allowed for the motion of the shell-1. Thus, we have to impose $\rho_{+}<r_{\min }$. Together with the condition $M_{2}=\mathcal{E}_{1} m_{1}>m_{1}$, the condition $\rho_{+}<r_{\min }$ leads to

$$
\mathcal{E}_{1}>1+\sqrt{1-\left(\frac{M_{2}}{Q_{2}}\right)^{2}}=1+(2 \epsilon)^{1 / 2}+\mathcal{O}(\epsilon) .
$$

If $\mathcal{E}_{1}$ is slightly larger than unity, the shell-1 shrinks to the radius less than $r_{\min }$.

The effective potential of the shell-1 at $r=r_{\min }$ is given by

$$
\begin{aligned}
V_{1}\left(r_{\text {min }}\right) & =1-\frac{1}{4}\left[\mathcal{E}_{1}+\frac{1}{(1+\epsilon)^{2} \mathcal{E}_{1}}\right]^{2} \\
& =-\frac{1}{4}\left(\mathcal{E}_{1}-\frac{1}{\mathcal{E}_{1}}\right)^{2}+\left(1+\frac{1}{\mathcal{E}_{1}^{2}}\right) \epsilon+\mathcal{O}\left(\epsilon^{2}\right) .
\end{aligned}
$$

If the shell- 1 is almost marginally bound, i.e., $\mathcal{E}_{1}=1+(2 \epsilon)^{1 / 2} \alpha$ with $\alpha$ larger than one but of order unity, we have

$$
V_{1}\left(r_{\min }\right)=-2\left(\alpha^{2}-1\right) \epsilon+\mathcal{O}\left(\epsilon^{2}\right)
$$

From the above result, we can see that, in the almost marginally bound case, the speed of

the shell-1 at $r=r_{\min }$, which is given by $\sqrt{-V_{1}\left(r_{\min }\right)}$, is very small. The high speed of the shell-1 at $r=r_{\min }$ is achieved only if $\mathcal{E}_{1}-1$ is almost equal to or larger than unity.

\section{B. The shell-2}

We assume that the shell-2 starts to fall inward from infinity at rest. This assumption is equivalent to the condition $\mathcal{E}_{2}=1$, i.e., $M_{3}-M_{2}=m_{2}$. As long as we see the effective 
potential $V_{2}, \mathcal{E}_{2}=-1$ seems to also be a solution, but it is not true due to Eq. (13): negative $\mathcal{E}_{2}$ implies the bound motion.

We adopt the following parametrization;

$$
\begin{aligned}
M_{3} & =M_{2}+\mu M_{2}=(1+\mu) M_{2}, \\
Q_{3} & =(1+q) Q_{2}=(1+\epsilon)(1+q) M_{2}, \\
m_{2} & =\mu M_{2} .
\end{aligned}
$$

Note that $\mu$ is positive since $m_{2}$ is positive.

By the careful analysis, we see that, in order that the shell-2 which is at rest at infinity starts to fall inward and collides with the shell-1 at $r=r_{\min }$, the two cases appear. In the first case, the following condition holds:

$$
0<\mu<q(1+\epsilon)+\epsilon
$$

for

$$
-\frac{\epsilon}{1+\epsilon}<q \leq 0
$$

By contrast, in the second case, the following condition holds:

$$
\mu_{\mathrm{m}}<\mu<q(1+\epsilon)+\epsilon
$$

for

$$
0<q<q_{*}
$$

where

$$
\begin{aligned}
& \mu_{\mathrm{m}}=(1+\epsilon)\left\{1+\epsilon-\sqrt{(1+\epsilon)^{2}-1}\right\}\left[\sqrt{1+\frac{q(q+2)}{\left\{1+\epsilon-\sqrt{(1+\epsilon)^{2}-1}\right\}^{2}}-1}\right] \\
& q_{*}=\frac{\epsilon^{2}+\sqrt{\epsilon(1+\epsilon)\left(2+\epsilon+\epsilon^{2}\right)}}{2(1+\epsilon)}
\end{aligned}
$$

The derivation of the above conditions is given in Appendix $B$, 


\section{The case with negative charge or without charge}

From the conditions (22), we obtain

$$
q=-\frac{\epsilon w_{-}}{1+\epsilon} \quad \text { with } \quad 0 \leq w_{-}<1
$$

Substituting the above expression into Eq. (21), we have

$$
0<\mu<\epsilon\left(1-w_{-}\right)
$$

Thus we get

$$
\mu=\epsilon\left(1-w_{-}\right) x_{-} \quad \text { with } 0<x_{-}<1 .
$$

By using the expressions (27) and (28), we have

$$
V_{2}\left(r_{\min }\right)=-\frac{\left[\left(1-w_{-}\right) x_{-}+w_{-}\right]^{2}}{\left(1-w_{-}\right)^{2} x_{-}^{2}}+\mathcal{O}(\epsilon)<0 .
$$

2. The case with positive charge

From the conditions (24), we obtain

$$
q=\left(\frac{\epsilon}{2}\right)^{1 / 2} w_{+} \quad \text { with } 0 \leq w_{+}<\epsilon^{-1 / 2} \sqrt{2} q_{*} .
$$

Note that $\left|\epsilon^{-1 / 2} \sqrt{2} q_{*}-1\right| \ll 1$. From Eq. (23), we have

$$
\mu=\mu_{\mathrm{m}}\left(1-x_{+}\right)+[q(1+\epsilon)+\epsilon] x_{+} \quad \text { with } 0<x_{+}<1 \text {. }
$$

Substituting Eq. (30) into the above expression, we have

$$
\mu=\left(\frac{\epsilon}{2}\right)^{1 / 2} w_{+}+\epsilon\left(w_{+}-w_{+} x_{+}+x_{+}\right)+\mathcal{O}\left(\epsilon^{3 / 2}\right)
$$

By using the expressions (30) and (31), we have

$$
V_{2}\left(r_{\text {min }}\right)=-\frac{2 \epsilon}{w_{+}^{2}} x_{+}\left(1-w_{+}\right)\left\{2 w_{+}+x_{+}\left(1-w_{+}\right)\right\}+\mathcal{O}\left(\epsilon^{3 / 2}\right)<0 .
$$

\section{The energy of collision}

We consider the collision of two shells such that the shell-1, which is initially ingoing, turns back as an outgoing shell and then collides with the ingoing shell-2 at $r=r_{\min }$. In this subsection, we estimate the center of mass energy for this collision. 


\section{The case with negative charge or without charge}

By using Eqs. (16), (20) and (29), we have

$$
E_{\mathrm{cm}}=\frac{M_{3}}{\mathcal{E}_{1}}\left[1+\left(\mathcal{E}_{1}^{2}-1\right)\left\{\left(1-w_{-}\right) x_{-}+w_{-}\right\}\right]^{1 / 2}+\mathcal{O}(\epsilon)
$$

The above equation implies that the collision energy of the shells at their center of mass frame does not exceed the total energy of the system $M_{3}$. However the gamma factor of the relative velocity between the shells can be very large:

$$
\Gamma \equiv-g_{\mu \nu} u_{1}^{\mu} u_{2}^{\nu}=\frac{1}{2 \epsilon}\left(\frac{\left(1-w_{-}\right) x_{-}+w_{-}}{\left(1-w_{-}\right) x_{-}}\right)\left(\frac{\mathcal{E}_{1}^{2}-1}{\mathcal{E}_{1}}\right)+\mathcal{O}\left(\epsilon^{0}\right),
$$

and hence $E_{\mathrm{P}}$ can also be very large. By using Eqs. (17), (201) and (29), we have

$$
\begin{aligned}
E_{\mathrm{P}} & =\frac{m}{\epsilon^{1 / 2}}\left(\frac{\left(1-w_{-}\right) x_{-}+w_{-}}{\left(1-w_{-}\right) x_{-}}\right)^{1 / 2}\left(\frac{\mathcal{E}_{1}^{2}-1}{\mathcal{E}_{1}}\right)^{1 / 2}+\mathcal{O}\left(\epsilon^{1 / 2}\right) \\
& \simeq 9.4 \times 10^{18}\left(\frac{\left(1-w_{-}\right) x_{-}+w_{-}}{\left(1-w_{-}\right) x_{-}}\right)^{1 / 2}\left(\frac{\mathcal{E}_{1}^{2}-1}{\mathcal{E}_{1}}\right)^{1 / 2}\left(\frac{m}{m_{\mathrm{p}}}\right)\left(\frac{10^{-38}}{\epsilon}\right)^{1 / 2} \mathrm{GeV}
\end{aligned}
$$

where $m_{\mathrm{p}}$ is the mass of a proton.

The above result implies that $E_{\mathrm{P}}$ can be indefinitely large in the limit of $\left(1-w_{-}\right) x_{-} \rightarrow 0$ with $\left[\left(1-w_{-}\right) x_{-}+w_{-}\right]^{1 / 2}$ fixed. However, we should note that the number of constituent particles of the shell $N \equiv m_{2} / m$ should be much larger than unity so that the continuum approximation is valid. By the definition of $N$, we have

$$
N=\epsilon(1-w) x \frac{M_{2}}{m}=1.2 \times 10^{19}\left(1-w_{-}\right) x_{-}\left(\frac{\epsilon}{10^{-38}}\right)\left(\frac{M_{2}}{M_{\odot}}\right)\left(\frac{m_{\mathrm{p}}}{m}\right),
$$

where $M_{\odot}$ is the solar mass. The above result implies that $\left(1-w_{-}\right) x_{-}$must not be too small so that $N$ is larger than unity. But our result still implies that, through the collision between two shells with charge of the different sign or with no charge, the energy of the collision between their constituent particles can exceed the Planck scale.

\section{The case with positive charge}

By using Eqs. (16), (20) and (32), we have

$$
E_{\mathrm{cm}}=\frac{M_{3}}{\mathcal{E}_{1}}\left[1+\frac{1}{2}\left(\mathcal{E}_{1}^{2}-1\right)\left\{\sqrt{w_{+}^{2}+V\left(w_{+}, x_{+}\right)}+\sqrt{V\left(w_{+}, x_{+}\right)}\right\}\right]^{1 / 2}+\mathcal{O}\left(\epsilon^{1 / 2}\right),
$$


where

$$
V(w, x):=x(1-w)[2 w+x(1-w)]
$$

The above equation implies that the collision energy of the shells at their center of mass frame does not exceed the total energy of the system $M_{3}$ also in the positive charge case. However, also in this case, since the gamma factor of the relative velocity between the shells can be very large, $E_{\mathrm{P}}$ can also be very large. By using Eqs. (17), (20) and (32), we have

$$
\begin{aligned}
E_{\mathrm{P}} & =\frac{m}{(2 \epsilon)^{1 / 4}}\left[\left(\mathcal{E}_{1}-\frac{1}{\mathcal{E}_{1}}\right) \frac{\sqrt{w_{+}^{2}+V\left(w_{+}, x_{+}\right)}+\sqrt{V\left(w_{+}, x_{+}\right)}}{w_{+}}\right]^{1 / 2}+\mathcal{O}\left(\epsilon^{1 / 4}\right) \\
& \simeq 7.9 \times 10^{18}\left[\left(\mathcal{E}_{1}-\frac{1}{\mathcal{E}_{1}}\right) \frac{\sqrt{w_{+}^{2}+V\left(w_{+}, x_{+}\right)}+\sqrt{V\left(w_{+}, x_{+}\right)}}{w_{+}}\right]^{1 / 2} \\
& \times\left(\frac{m}{m_{\mathrm{p}}}\right)\left(\frac{10^{-76}}{\epsilon}\right)^{1 / 4} \mathrm{GeV} .
\end{aligned}
$$

In this case, the fine tuning of $\epsilon$ required for the ultra-high energy collision is severer than the negative charge or neutral case.

The above result implies that $E_{\mathrm{P}}$ can be indefinitely large in the limit of $w_{+} \rightarrow 0$ with $x_{+}$ fixed. However, we should note that we have assumed that $\epsilon^{1 / 2} w_{+} \gg \epsilon x_{+}$, i.e., $w_{+} \gg \epsilon^{1 / 2} x_{+}$, when we derived Eq. (31). Thus, in Eq. (35), such a limit must not be taken. But our result still implies that, through the collision between two shells with the same sign of charge, the energy of the collision between their constituent particles can exceed the Planck scale.

\section{SUMMARY AND DISCUSSION}

We studied a collision between two concentric spherical thin dust shells: the inner shell is over-charged, whereas the outer one may or may not be charged. The domain in the inner shell is assumed to be described by the Minkowskian geometry, whereas the domain between the two shells and the domain outside the outer shell are assumed to be described by the over-charged Reissner-Nordström geometry. First, the inner shell starts to fall inwards from infinity with finite initial velocity and eventually turns outward due to its self-electric force which overcomes its self-gravitational force. The outer shell starts to fall inward from infinity with vanishing initial velocity after a sufficient time after the inner shell has left. It will collide with inner shell going outward. 
We found that if the Reissner-Nordström geometry between these shells is almost extreme but a bit over-charged, the energy of collision between two of constituent particles of these shells at their center of mass frame can be much larger than the Planck scale even if the mass of particle is order of proton mass, whereas the energy of collision between two shells at their center of mass frame can not exceed the total energy of the system. We would like to stress that neither black hole horizon nor naked singularity is necessary to achieve very high energy of the collision between constituent particles of the shells in this case. The necessary condition to achieve the high energy is that the collision occurs at $r=r_{\min } \equiv Q_{2}^{2} / M_{2}$, where $Q_{2}$ and $M_{2}$ are the charge and mass parameters of the Reissner-Nordström geometry between these shells.

It is important fact that the inner over-charged shell can shrink to $r<r_{\min }$ if the initial inward velocity of the inner shell at infinity exceed a small threshold value. Furthermore, the inner shell can shrink to an arbitrarily small radius: we can see from Eq. (19) that, in the limit $\mathcal{E}_{1} \rightarrow \infty$, the radius of the turning point $r=\rho_{+}$vanishes. This means that even if no naked singularity forms, a domain described by the over-charged Reissner-Nordström geometry arbitrarily close to the naked singularity may appear as a transient phenomenon. The physical phenomena similar to the PJ process may occur in such a domain, even though no naked singularity forms.

As mentioned, the infinitesimally thin shell is a technical simplification. Although the stress-energy tensor of the shell diverges, this prescription is based on the assumption that the self-gravity of each constituent particle of the thin shells is negligible: the local dynamics of each particle is described in the framework of the special relativity. However, if the collision energy of the particles at the center of mass frame exceeds the Planck scale, the colliding particles will make the spacetime highly curved, and black holes may form. Their motions after ultra-high energy collision cannot be described in the framework of the special relativity. This means that the motion of the shells after the collision is highly non-trivial problem, but this issue is out of the scope of this article.

\section{Acknowledgments}

MK is supported by the JSPS Grant-in-Aid for Scientific Research No.23.2182. KN thanks H. Ishihara and colleagues in the group of elementary particle physics and gravity 
at Osaka City University for useful discussions.

\section{Appendix A: The shell-1}

Here, we show that $\mathcal{E}_{1}$ must be larger than unity and the allowed domain for the motion of the shell-1 is restricted to $r \geq \rho_{+}$, where $\rho_{+}$is given in Eq. (19). As mentioned, $\mathcal{E}_{1}$ should be positive, and an inequality $0<M_{2}<Q_{2}$ holds.

First, we note that there is only one root of the equation $d V_{1}(r) / d r=0$ : the root is given by

$$
r=\rho_{\mathrm{m}} \equiv \frac{Q_{2}^{2}-m_{1}^{2}}{2 \mathcal{E}_{1} m_{1}}=\frac{1}{2 \mathcal{E}_{1} m_{1}}\left(Q_{2}^{2}-\frac{M_{2}^{2}}{\mathcal{E}_{1}^{2}}\right) .
$$

We can see that $\rho_{\mathrm{m}}$ is positive if and only if $\mathcal{E}_{1}$ is larger than $M_{2} / Q_{2}$ which is less than unity. Hence, in the case of $\mathcal{E}_{1}>M_{2} / Q_{2}, V_{1}(r)$ has one extremum at $r=\rho_{\mathrm{m}}$. We can easily see that this extremum is the maximum and $V_{1}\left(\rho_{\mathrm{m}}\right)=1$. Here note that $V_{1}(r) \rightarrow 1-\mathcal{E}_{1}^{2}$ in the limit $r \rightarrow \infty$. Hence, in the case of $0<\mathcal{E}_{1}<1$, the spatial asymptotic region is a domain forbidden for the motion of the shell-1. Since $V_{1}(r)$ is monotonically decreasing in the domain of $r>\rho_{\mathrm{m}}$ in the case of $\mathcal{E}_{1}=1$, we can see $V_{1}(r)>0$ in the domain of $r \geq \rho_{\mathrm{m}}$. Thus the spatial asymptotic region is the forbidden domain also in the case of $\mathcal{E}_{1}=1$. Only in the case of $\mathcal{E}_{1}>1$ the spatial asymptotic domain is allowed for the motion of the shell-1.

\section{1. $0<\mathcal{E}_{1}<M_{2} / Q_{2}$}

In this case, $V_{1}(r)$ is a monotonically increasing function of $r$, and only $r=\rho_{+}$is a positive root of $V_{1}(r)=0$. $V_{1}(r) \geq 0$ in the domain $r \geq \rho_{+}$, whereas $V_{1}(r)<0$ in the domain of $r<\rho_{+}$. Since $V_{1}\left(\rho_{1}\right)>0$ by Eq. (15), we find that $\rho_{1}>\rho_{+}$, and hence even if $V_{1}(r) \leq 0$ in the domain $r \leq \rho_{+}$, the domain $r \leq \rho_{+}$cannot be allowed for the motion of the shell-1 by Eq. (11). As a result, in the case of $0<\mathcal{E}_{1}<M_{2} / Q_{2}$, there is no domain allowed for the motion of the shell-1.

\section{2. $\quad \mathcal{E}_{1}=M_{2} / Q_{2}$}

There is no allowed domain for the motion of the shell-1, since $V_{1}$ is identically equal to $1-\left(M_{2} / Q_{2}\right)^{2}$ which is positive. 
3. $M_{2} / Q_{2}<\mathcal{E}_{1} \leq 1$

Only $r=\rho_{-}$is a positive root of $V_{1}(r)=0$. Since $V_{1}(r) \geq 0$ in the domain $r \geq \rho_{-}$, whereas $V_{1}(r)<0$ in the domain $r<\rho_{-}$, the situation of this case is very similar to the case of $0<\mathcal{E}_{1}<M_{2} / Q_{2}$. By the similar argument to the case of $0<\mathcal{E}_{1}<M_{2} / Q_{2}$, we can see that there is no allowed domain for the motion of the shell-1 in the case of $M_{2} / Q_{2}<\mathcal{E}_{1} \leq 1$.

\section{4. $\mathcal{E}_{1}>1$}

Both $r=\rho_{+}$and $r=\rho_{-}$are positive roots of $V_{1}(r)=0$. In this case, $V_{1}(r) \leq 0$ in the

domain $r \leq \rho_{-}$or $r \geq \rho_{+}$, whereas $V_{1}(r)>0$ in the domain $\rho_{-}<r<\rho_{+}$. We find from Eq. (15) that $\rho_{-}<\rho_{1}<\rho_{+}$holds, and hence the domain $r<\rho_{-}$is forbidden for the motion of the shell-1 by Eq. (111). The allowed domain for the motion of the shell-1 is only $r \geq \rho_{+}$.

In summary, the shell-1 is necessarily unbound $\mathcal{E}_{1}>1$ and the allowed domain is $r \geq \rho_{+}$, as long as we assume $0<M_{2}<Q_{2}$.

\section{Appendix B: The shell-2}

In this appendix, we derive the conditions (21) - (26) .

\section{From the over-charge condition}

Since we assume that the domain $D_{3}$ is described by the over-charged Reissner-Nordström geometry $Q_{3}>M_{3}$, we have

$$
\mu<(1+\epsilon) q+\epsilon
$$

Since $\mu$ is positive, $(1+\epsilon) q+\epsilon$ should be positive so that the above inequality has a solution. Hence, we have

$$
q>-\frac{\epsilon}{1+\epsilon}
$$




\section{From the marginally-bound condition}

In the marginally bound case, the effective potential $V_{2}$ of the shell- 2 is written in the form

$$
V_{2}(r)=-\frac{M_{2}}{\mu r} A_{1}(\mu ; q)-\left(\frac{M_{2}}{2 \mu r}\right)^{2} A_{2}(\mu ; q)
$$

where

$$
\begin{aligned}
& A_{1}(\mu ; q) \equiv \mu(\mu+2)-(1+\epsilon)^{2} q(q+2) \\
& A_{2}(\mu ; q) \equiv[(1+\epsilon) q-\mu][(1+\epsilon) q+\mu][(1+\epsilon)(q+2)-\mu][(1+\epsilon)(q+2)+\mu] .
\end{aligned}
$$

Since $V_{2}(r)$ should be negative in the domain allowed for the motion of the shell-2, the following condition should be satisfied so that the shell-2 can start to fall inward from infinity;

$$
A_{1}(\mu ; q)>0
$$

is satisfied, or both $A_{1}=0$ and $A_{2}>0$ hold simultaneously.

From Eq. (B3), we obtain the following constraint on $\mu$; in the case of $q(q+2)>0$,

$$
\mu>b
$$

should hold, where $b$ is a larger root of $A_{1}(b ; q)=0$ :

$$
b=-1+\sqrt{1+(1+\epsilon)^{2} q(q+2)},
$$

whereas, in the case of $q(q+2) \leq 0$, Eq. (B33) necessarily holds, since $\mu$ is positive.

The solution of the inequality $q(q+2) \leq 0$ is $-2 \leq q \leq 0$. However, since $-\epsilon(1+\epsilon)^{-1}$ is larger than -2 for $\epsilon>0$, we should consider from Eq. (B2) that the solution of the inequality $q(q+2) \leq 0$ is $-\epsilon(1+\epsilon)^{-1}<q \leq 0$.

The solution of the inequality $q(q+2)>0$ is $q<-2$ or $0<q$. However, Eq. (B2) implies that $q$ must be larger than -2 , and hence we should consider that the solution of the inequality $q(q+2)>0$ is $q>0$. In the case of $q(q+2)>0, b$ must be less than $(1+\epsilon) q+\epsilon$ so that Eqs. (B1) and (B4) are consistent with each other. By the definition of $b$, we have

$$
(1+\epsilon) q+\epsilon-b=(1+\epsilon)(1+q)-\sqrt{(1+\epsilon)^{2}(1+q)^{2}-\epsilon(2+\epsilon)} .
$$

We can see from the above equation that $(1+\epsilon) q+\epsilon-b>0$ is satisfied because of $q>0$. Hence, the consistency between Eqs. (B1) and (Bי 
In the case of $q(q+2)>0$, i.e., $q>0, A_{1}$ vanishes only for $\mu=b$. In this case, we can see

$$
\begin{aligned}
q(1+\epsilon)-b & <0, \\
q(1+\epsilon)+b & >0, \\
(q+2)(1+\epsilon)-b & >0, \\
(q+2)(1+\epsilon)+b & >0 .
\end{aligned}
$$

First, we prove Eq. (B5). We have

$$
q(1+\epsilon)-b=q(1+\epsilon)+1-\sqrt{1+(1+\epsilon)^{2} q(q+2)} .
$$

Since $q>0$, we can see

$$
[q(1+\epsilon)+1]^{2}-\left[1+(1+\epsilon)^{2} q(q+2)\right]=-2 \epsilon(1+\epsilon) q<0
$$

Hence, we obtain Eq. (B5).

Next, we prove Eq. (B6). We have

$$
q(1+\epsilon)+b=q(1+\epsilon)-1+\sqrt{1+(1+\epsilon)^{2} q(q+2)}
$$

Since $q>0$, we have

$$
[q(1+\epsilon)-1]^{2}-\left[1+(1+\epsilon)^{2} q(q+2)\right]=-2(2+\epsilon)(1+\epsilon) q<0 .
$$

Hence, we have Eq. (B6).

By the over-charge condition (B1), i.e., $q(1+\epsilon)+\mu>-\epsilon$, we can see that Eqs. (B7) and (B8) should be satisfied;

$$
\begin{aligned}
& (q+2)(1+\epsilon)-b=q(1+\epsilon)-b+2(1+\epsilon)>-\epsilon+2(1+\epsilon)=2+\epsilon>0, \\
& (q+2)(1+\epsilon)+b=q(1+\epsilon)-b+2 b>2+\epsilon+2 b>0 .
\end{aligned}
$$

Equations (B5) - (B8) imply that $A_{2}$ is negative, and thus this case is not of our interest.

In summary, we have found that the over-charge condition (B1) and marginally bound

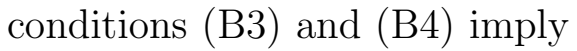

$$
\begin{aligned}
& 0<\mu<(1+\epsilon) q+\epsilon \quad \text { for } \quad-\frac{\epsilon}{1+\epsilon}<q \leq 0, \\
& b<\mu<(1+\epsilon) q+\epsilon \text { for } \quad 0<q .
\end{aligned}
$$




\section{To reach $r=r_{\min }$}

In order that a collision can occur at $r=r_{\text {min }}$, we have

$$
F_{2}\left(r_{\min }\right)=-(1+\epsilon)^{-4} G(\mu ; q)>0
$$

and

$$
V_{2}\left(r_{\min }\right)=-\frac{1}{4 \mu^{2}}(1+\epsilon)^{-4} H(\mu ; q) \leq 0
$$

where

$$
\begin{aligned}
& G(\mu ; q)=\mu^{2}-2(1+\epsilon)^{2} \mu+(1+\epsilon)^{2} q(q+2) \\
& H(\mu ; q)=\left[\mu^{2}+2(1+\epsilon)^{2} \mu-(1+\epsilon)^{2} q(q+2)\right]^{2}-4 \epsilon(2+\epsilon)(1+\epsilon)^{2} \mu^{2} .
\end{aligned}
$$

First, we consider the condition (B11). The condition (B11) is rewritten in the form of $G(\mu ; q)<0$. It can be easily seen that $q(q+2)$ should be less than $(1+\epsilon)^{2}$ so that this inequality has a solution with respect to $\mu$. If $q(q+2) \leq 0$, i.e., $-\epsilon(1+\epsilon)^{-1}<q \leq 0$ holds, this constraint is satisfied. On the other hand, if $q(q+2)>0$, i.e., $q>0$ holds, the constraint $q(q+2)<(1+\epsilon)^{2}$ leads to

$$
0<q<\sqrt{1+(1+\epsilon)^{2}}-1
$$

Then, we obtain

$$
\begin{aligned}
& 0<\mu<b_{+} \quad \text { for } \quad-\frac{\epsilon}{1+\epsilon}<q \leq 0, \\
& b_{-}<\mu<b_{+} \quad \text { for } \quad 0<q<\sqrt{1+(1+\epsilon)^{2}}-1 \text {, }
\end{aligned}
$$

where $b_{ \pm}$are the roots of $G\left(b_{ \pm} ; q\right)=0$ :

$$
b_{ \pm}=(1+\epsilon)^{2} \pm(1+\epsilon) \sqrt{(1+\epsilon)^{2}-q(q+2)}
$$

In order to see which are sharp conditions, the pair of Eqs. (B91) and (B10) or the pair of Eqs. ( $(\overline{\mathrm{B} 15})$ and $(\underline{\mathrm{B} 16})$, we investigate which is larger, $b_{+}$or $q(1+\epsilon)+\epsilon$. We have

$$
q(1+\epsilon)+\epsilon-b_{+}=q(1+\epsilon)-\left(1+\epsilon+\epsilon^{2}\right)-(1+\epsilon) \sqrt{(1+\epsilon)^{2}-q(q+2)} .
$$

Here we define the following quantity

$$
c \equiv q(1+\epsilon)-\left(1+\epsilon+\epsilon^{2}\right)
$$


If $c$ is negative, the quantity $q(1+\epsilon)+\epsilon-b_{+}$is also negative. We can easily see that $c$ is negative in the case of $-\epsilon(1+\epsilon)^{-1}<q \leq 0$. By contrast, in the case of $0<q<$ $\sqrt{1+(1+\epsilon)^{2}}-1$, we have

$$
\begin{aligned}
c & <\left[\sqrt{1+(1+\epsilon)^{2}}-1\right](1+\epsilon)-\left(1+\epsilon+\epsilon^{2}\right) \\
& =(1+\epsilon) \sqrt{1+(1+\epsilon)^{2}}-\left[1+(1+\epsilon)^{2}\right] .
\end{aligned}
$$

We can see

$$
\left[(1+\epsilon) \sqrt{1+(1+\epsilon)^{2}}\right]^{2}-\left[1+(1+\epsilon)^{2}\right]^{2}=-\left[1+(1+\epsilon)^{2}\right]<0 .
$$

Hence, $c$ is negative also in the case of $0<q<\sqrt{1+(1+\epsilon)^{2}}-1$. Thus, we obtain

$$
q(1+\epsilon)+\epsilon<b_{+} \quad \text { for } \quad-\frac{\epsilon}{1+\epsilon}<q<\sqrt{1+(1+\epsilon)^{2}}-1 .
$$

Now, from the over-charge condition (B1), the marginally-bound condition (B33) and (B4), the constraint (B11), we have

$$
\begin{array}{lll}
0<\mu<q(1+\epsilon)+\epsilon & \text { for } \quad-\frac{\epsilon}{1+\epsilon}<q \leq 0, \\
\max \left[b_{-}, b\right]<\mu<q(1+\epsilon)+\epsilon & \text { for } \quad 0<q<\sqrt{1+(1+\epsilon)^{2}}-1 .
\end{array}
$$

Here, let us focus on the condition (B18). In order that Eq. (B18) is satisfied, $b_{-}$must be less than $q(1+\epsilon)+\epsilon$. Thus, we have

$$
q(1+\epsilon)+\epsilon-b_{-}=c+(1+\epsilon) \sqrt{(1+\epsilon)^{2}-q(q+2)}>0 .
$$

Since $c$ is negative, $|c|$ should be less than $(1+\epsilon) \sqrt{(1+\epsilon)^{2}-q(q+2)}$. Thus we have

$$
c^{2}-\left[(1+\epsilon) \sqrt{(1+\epsilon)^{2}-q(q+2)}\right]^{2}=2(1+\epsilon)^{2} q^{2}-2 \epsilon^{2}(1+\epsilon) q-\epsilon\left(2 \epsilon^{2}+3 \epsilon+2\right)<0 .
$$

In order that the above inequality folds, $q$ should satisfy

$$
0<q<q_{*},
$$

where

$$
q_{*}:=\frac{\epsilon^{2}+\sqrt{\epsilon(1+\epsilon)\left(2+\epsilon+\epsilon^{2}\right)}}{2(1+\epsilon)} .
$$

Since we are interested in the case of $0<\epsilon \ll 1$, we have $q_{*}=\sqrt{\epsilon / 2}+\mathcal{O}\left(\epsilon^{3 / 2}\right)<$ $\sqrt{1+(1+\epsilon)^{2}}-1$. In order to know which is larger, $b$ or $b_{-}$, we should again note that 
$b$ is the larger root of $A_{1}(b ; q)=0$, whereas $b_{ \pm}$are the roots of $G\left(b_{ \pm} ; q\right)=0$ with $b_{+}>b_{-}$. We should also note that there is only one root of the equation $A_{1}(\mu ; q)=G(\mu ; q)$ : it is given by

$$
\mu=b_{\mathrm{m}}:=\frac{(1+\epsilon)^{2} q(q+2)}{1+(1+\epsilon)^{2}} .
$$

The function $A_{1}(\mu ; q)$ takes a minimum value at $\mu=-1$, whereas the function $G(\mu ; q)$ takes a minimum value at $\mu=(1+\epsilon)^{2}$. Thus, we have $-1<b_{\mathrm{m}}, b, b_{-}<(1+\epsilon)^{2}$. From these facts, we can see that $b<b_{\mathrm{m}}<b_{-}$for $A_{1}\left(b_{\mathrm{m}} ; q\right)=G\left(b_{\mathrm{m}} ; q\right)>0, b=b_{\mathrm{m}}=b_{-}$for $A_{1}\left(b_{\mathrm{m}} ; q\right)=G\left(b_{\mathrm{m}} ; q\right)=0$, and $b>b_{\mathrm{m}}>b_{-}$for $A_{1}\left(b_{\mathrm{m}} ; q\right)=G\left(b_{\mathrm{m}} ; q\right)<0$. We have

$$
A_{1}\left(b_{\mathrm{m}} ; q\right)=G\left(b_{\mathrm{m}} ; q\right)=\frac{(1+\epsilon)^{2} q(q+2)}{\left[1+(1+\epsilon)^{2}\right]^{2}}\left[(1+\epsilon)^{2} q(q+2)-\left\{(1+\epsilon)^{4}-1\right\}\right] .
$$

From the above equation, we can see that $A_{1}\left(b_{\mathrm{m}} ; q\right)=G\left(b_{\mathrm{m}} ; q\right)>0$ for $q>q_{2}, A_{1}\left(b_{\mathrm{m}} ; q\right)=$ $G\left(b_{\mathrm{m}} ; q\right)=0$ for $q=q_{2}$, and $A_{1}\left(b_{\mathrm{m}} ; q\right)=G\left(b_{\mathrm{m}} ; q\right)<0$ for $0<q<q_{2}$ (note that we focus on the case of $q>0$.), where

$$
q_{2}:=1+\frac{\sqrt{(1+\epsilon)^{4}+(1+\epsilon)^{2}-1}}{1+\epsilon} .
$$

Hence, we have

$$
\begin{aligned}
& b>b_{-} \quad \text { for } 0<q<q_{2} \text {, } \\
& b=b_{-} \quad \text { for } \quad q=q_{2}, \\
& b<b_{-} \quad \text { for } \quad q>q_{2} \text {, }
\end{aligned}
$$

In the case of $0<\epsilon \ll 1$, we have $q_{2}=2+\mathcal{O}(\epsilon)$ and hence we find that $q_{*}<q_{2}$. This implies that $b>b_{-}$holds for $0<q<q_{*}$. Thus, Eq. (B18) should be replaced by

$$
b<\mu<q(1+\epsilon)+\epsilon \quad \text { for } \quad 0<q<q_{*} .
$$

Next, we consider the condition $(\overline{\mathrm{B} 12})$. In order that $r=r_{\min }$ is in the allowed domain for the motion of the shell-2, $H(\mu ; q)$ should be non-negative. This condition is written in the form

$$
|I(\mu ; q)|>2 \mu(1+\epsilon) \sqrt{(1+\epsilon)^{2}-1}
$$

where

$$
I(\mu ; q):=\mu^{2}+2(1+\epsilon)^{2} \mu-(1+\epsilon)^{2} q(q+2) .
$$




\section{a. The case with negative charge or without charge}

In this case, the condition (B17) leads to $q(q+2)<0$. Then, since $\mu>0$, we can easily see $I(\mu ; q)>0$. The condition (

$$
\mu^{2}+2(1+\epsilon)\left[1+\epsilon-\sqrt{(1+\epsilon)^{2}-1}\right]-(1+\epsilon)^{2} q(q+2)>0
$$

The above condition is always satisfied under the condition $(\overline{\mathrm{B} 17})$, since $q(q+2)<0$ and $\mu>0$. As a result, the sphere $r=r_{\text {min }}$ is in the allowed domain for the motion of the shell-2 under the condition (B17).

\section{b. The case with positive charge}

We consider the case of $q>0$, and Eq. (B19) should be satisfied. Here note again that $A_{1}(b ; q)=0$, and hence we can easily see that

$$
H(b ; q)=-4 b^{2} \epsilon(2+\epsilon)<0 .
$$

Hence, in the case of $\mu=b$, the shell-2 cannot arrive at $r=r_{\min }$. We have

$$
\begin{array}{ll}
I(\mu ; q) \leq 0 & \text { for } \quad d_{-} \leq \mu \leq d_{+} \\
I(\mu ; q)>0 & \text { for } \mu<d_{-}, \quad d_{+}<\mu
\end{array}
$$

where $d_{ \pm}$are the roots of $I\left(d_{ \pm} ; q\right)=0$ :

$$
d_{ \pm}=-(1+\epsilon)^{2} \pm \sqrt{(1+\epsilon)^{4}+(1+\epsilon)^{2} q(q+2)}
$$

Since $d_{-}<0<b$ and $f(b)+2 b \epsilon(2+\epsilon)>0$, we have $d_{-}<0<d_{+}<b$. This means that $I(\mu ; q)>0$ for $\mu>b$. Thus, the condition (Bי $)$ leads to

$$
\begin{aligned}
J(\mu ; q) & :=I(\mu ; q)-2 \mu(1+\epsilon) \sqrt{(1+\epsilon)^{2}-1} \\
& =A_{1}(\mu ; q)-2 \mu\left[1+\epsilon-\sqrt{(1+\epsilon)^{2}-1}\right] \sqrt{(1+\epsilon)^{2}-1}>0
\end{aligned}
$$

Together with $\mu>0$, the above condition leads to $\mu>\mu_{\mathrm{m}}$, where

$$
\mu_{\mathrm{m}}:=(1+\epsilon)\left\{1+\epsilon-\sqrt{(1+\epsilon)^{2}-1}\right\}\left[\sqrt{1+\frac{q(q+2)}{\left\{1+\epsilon-\sqrt{(1+\epsilon)^{2}-1}\right\}^{2}}}-1\right]
$$


Since $A_{1}(q(1+\epsilon)+\epsilon ; q)=\epsilon(2+\epsilon)$, we have

$$
\begin{aligned}
J(q(1+\epsilon)+\epsilon ; q) & =\epsilon\left[(1+2 \epsilon)(2+\epsilon)-2(1+\epsilon) \sqrt{(1+\epsilon)^{2}-1}\right] \\
& -2 q(1+\epsilon)\left[1+\epsilon-\sqrt{(1+\epsilon)^{2}-1}\right] \sqrt{(1+\epsilon)^{2}-1}
\end{aligned}
$$

From the above equation and Eq. (B27), we obtain

$$
q<q_{2}:=\frac{\epsilon\left[(1+2 \epsilon)(2+\epsilon)-2(1+\epsilon) \sqrt{(1+\epsilon)^{2}-1}\right]}{2\left[1+\epsilon-\sqrt{(1+\epsilon)^{2}-1}\right] \sqrt{(1+\epsilon)^{2}-1}}=\left(\frac{\epsilon}{2}\right)^{1 / 2}\left(1+\frac{3}{4} \epsilon+\mathcal{O}\left(\epsilon^{3 / 2}\right)\right) .
$$

Since $q_{*}=(\epsilon / 2)^{1 / 2}\left[1-\epsilon / 4+\mathcal{O}\left(\epsilon^{3 / 2}\right)\right]$, we have $q_{2}>q_{*}$. Hence, instead of Eq. (B19), the following condition should hold;

$$
\mu_{\mathrm{m}}<\mu<(1+\epsilon) q+\epsilon \quad \text { for } \quad 0<q<q_{*} \text {. }
$$

[1] M. Banados, J. Silk, S.M. West, Phys. Rev. Lett. 103, 111102 (2009).

[2] M. Banados, B. Hassanain, J. Silk, S. M. West, Phys. Rev. D 83, 023004 (2011); A. Williams, arXiv/astro.ph/1101.4819 (2011).

[3] E. Berti, V. Cardoso, L. Gualtieri, F. Pretorius, U. Sperhake, Phys. Rev. Lett. 103, 239001 (2009); T. Jacobson, T. P. Sotiriou, Phys. Rev. Lett. 104, 021101 (2010).

[4] K.S. Thorne, Astrophys. J. 191, 507(1974).

[5] T. Harada, H. Nemoto, U. Miyamoto, Phys. Rev. D 86024027 (2012), Erratum-ibid. D 86 069902 (2012); T. Harada, M. Kimura, Phys. Rev. D 83, 084041 (2011); Phys. Rev. D 83, 024002 (2011). A.A. Grib, Y.V. Pavlov, Astropart. Phys. 34, 581 (2011); arXiv:1004.0913; arXiv:1007.3222; K. Lake, Phys. Rev. Lett. 104, 211102 (2010); Grav. Cosmol. 17, 42 (2011); O.B. Zaslavskii, Phys. Rev. D 84,024007 (2011); Class. Quant. Grav. 28, 105010 (2011); JETP Lett. 92, 571 (2010); Phys. Rev. D 82, 083004 (2010); S. Gao, C. Zhong, arXiv:1106.2852; J. Said, K.Z. Adami, Phys. Rev. D 83, 104047 (2011); C. Liu, S. Chen, J. Jing, arXiv:1104.3225; Y. Zhu, S. Wu, Y. Liu, Y. Jiang, arXiv:1103.3848; C. Liu, S. Chen, C. Ding, J. Jing, arXiv:1012.5126; Y. Li, J. Yang, Y-L. Li, S. Wei, Y. Liu, arXiv:1012.0748; P. Mao, R. Li, L. Jia, J. Ren, arXiv:1008.2660; S. Wei, Y. Liu, H. Li, F. Chen, JHEP 1012, 066 (2010); S. Wei, Y. Liu, H. Guo, C. Fu, Phys. Rev. D 82, 103005 (2010); W. Yao, S. Chen, C. Liu, J. Jing, arXiv:1105.6156. 
[6] M. Patil, P. Joshi, arxiv/1103.1082(2011); arxiv/1103.1083(2011).

[7] R. Penrose, Riv. Nuovo Cim 1, 252 (1969) [Gen. Rel. Grav. 34, 1141 (2002)].

[8] O. Zaslavskii, JETP Lett. 92, 571(2010).

[9] M. Kimura, K. Nakao, H. Tagoshi, Phys. Rev. D 83, 044013 (2011).

[10] M. Patil, P. Joshi, M. Kimura, K. Nakao, Phys. Rev. D 86084023 (2012).

[11] S.W. Hawking, G.F.R. Ellis, "Large scale structure of space-time", Cambridge University Press, Cambridge (1973).

[12] W. Israel, Nuovo Cimento B 44, 1 (1966); 463(E) (1967); Nuovo Cimento B 44, 1(1966).

[13] E. Poisson, "Relativist's Toolkit, The mathematics of black hole mechanics", Cambridge University Press, Cambridge (2004).

[14] C.W. Misner and D.H. Sharp, Phys.Rev. 136, B571 (1964).

[15] H. Kodama, Prog. Theor. Phys. 63, 1217 (1980).

[16] S.A. Hayward, Phys. Rev. D 53. 193 (1996).

[17] As already mentioned, Eq. (44) implies $f_{A+1}<f_{A}$, and this condition is rewritten in the form $\mathcal{M}_{A+1}(r)-\mathcal{M}_{A}(r)>0$, where $\mathcal{M}_{A}$ is equal to the so called Misner-Sharp mass of this system, $\mathcal{M}_{A}(r)=M_{A}-Q_{\Lambda}^{2} / 2 r$. The Misner-Sharp mass $\mathcal{M}(r)$ has a meaning of the energy within a sphere of radius $r$ 14 16$]$. Hence $\mathcal{M}_{A+1}(r)-\mathcal{M}_{A}(r)$ is regarded as the energy of the shell- $A$ of radias $r$ and is necessarily positive as expected, even if $M_{A+1}-M_{A}$ is negative.

[18] Here it should be noted that Eq. (6) is derived from not only Eq. (5) but also

$$
\sqrt{\dot{r}^{2}+f_{A+1}(r)}=-\sqrt{\dot{r}^{2}+f_{A}(r)}+\frac{m_{A}}{r}
$$

The above equation can be satisfied only if the shell exits in a black hole domain, i.e., $f_{A+1}<0$, since $\dot{t}_{A+1}$ can be negative there. By the similar argument as in the case of Eq. (4), the above equation leads to the following inequalities:

$$
\begin{array}{rlrl}
r & \leq \rho_{A} & & \text { for } \quad \mathcal{E}_{A}>0, \\
Q_{A+1}^{2}-Q_{A}^{2}+m_{A}^{2} & \geq 0 \quad & \text { for } \quad \mathcal{E}_{A}=0, \\
r & \geq \rho_{A} \quad & \text { for } \quad \mathcal{E}_{A}<0 .
\end{array}
$$

In the case of $\mathcal{E}_{A}>0, Q_{A+1}^{2}-Q_{A}^{2}+m_{A}^{2}$ should be positive so that Eq. (B29) is satisfied. 